\title{
Bone Mineral Density in Children Treated With Daily or Periodical Inhaled Budesonide: The Helsinki Early Intervention Childhood Asthma Study
}

\author{
MARKKU TURPEINEN, ANNA S. PELKONEN, KURT NIKANDER, RITVA SORVA, OLOF SELROOS, \\ KAISU JUNTUNEN-BACKMAN, AND TARI HAAHTELA
}

\author{
Department of Allergy [M.T., A.S.P., R.S., K.J.-B., T.H.], Helsinki University Central Hospital, FIN-00250 Helsinki, Finland; AstraZeneca \\ $R \& D$ [K.N.], SE-221 85 Lund, Sweden; Semeco AB [O.S.], SE-260 83 Vejbystrand, Sweden
}

\begin{abstract}
In a double-blind, randomized study, 136 children, 5-10-y-old, with newly detected persistent asthma received budesonide (BUD) $400 \mu \mathrm{g}$ twice daily for $1 \mathrm{mo}$ and thereafter $200 \mu \mathrm{g}$ twice daily for 5 mo. Thereafter, 50 children were treated with BUD $100 \mu \mathrm{g}$ twice daily, whereas 44 children used BUD as needed for $1 \mathrm{y}$; an additional 42 children received disodium cromoglycate (DSCG). Asthma exacerbations were treated with BUD for 2 wk in a dose of $400 \mu \mathrm{g}$ twice daily in all groups. In this secondary analysis, bone mineral density (BMD) of the lumbar vertebrae was measured before and after the 18-mo treatment. Compared with DSCG, regular BUD treatment resulted in a significantly smaller increase in BMD $(0.023$ versus $\left.0.034 \mathrm{~g} / \mathrm{cm}^{2} ; p=0.023\right)$ and height $(7.75$ versus $8.80 \mathrm{~cm} ; p=$ $0.001)$. Periodic treatment did not affect BMD. No intergroup differences were observed when BMD data were adjusted for changes in height. Daily BUD treatment in prepubertal children may slow down the increment in BMD and standing height. This was not observed in children receiving BUD periodically after the initial regular BUD treatment. The correlation between height and BMD suggests that following children's height might afford an estimation of inhaled corticosteroid effects on bone. (Pediatr Res 68: 169-173, 2010)
\end{abstract}

$\mathrm{I}_{\mathrm{t}}^{\mathrm{n}}$ nhaled corticosteroids (ICS) are recommended first-line treatment for all patients with persistent asthma (1). Systemic exposure to corticosteroids can suppress bone formation and increase bone resorption resulting in loss of bone mass and risk of fractures (2). Also, in children, the use of oral steroids has been associated with a dose-dependent increase in fracture risk (3). Case-control studies, too, have identified an increased risk of fractures with high doses of $\operatorname{ICS}(4,5)$, particularly in elderly patients (6). Therefore, there has been some concern that the use of ICS as long-term asthma maintenance therapy in children could have a detrimental effect on bone mineral density (BMD) and risk of fractures.

A recent Cochrane systematic review found that low to moderate doses of ICS did not affect BMD or risk of fractures in adults (7). However, it is not possible to extrapolate findings from adults to children. Bone mass in children is also influenced by

Received November 4, 2009; accepted April 28, 2010.

Correspondence: Markku Turpeinen, MD, PhD, Skin and Allergy Hospital, Department of Allergy, Helsinki University Hospital, Meilahdentie 2, FIN-00250 Helsinki, Finland; e-mail: markku.t.turpeinen@hus.fi

Supported by the Helsinki University Central Hospital (Grant TYH 2303) and AstraZeneca, Lund, Sweden. height, age, race, exercise, presence of chronic illnesses, and especially the stage of puberty (8).

The clinically most important outcome for the effects of ICS on bone is an increased risk of fractures. This is not easy to study, particularly, in children in randomized prospective trials. The use of biochemical markers is restricted to describe bone metabolism, but BMD has been used as a surrogate marker of an increased susceptibility to fractures. In this respect, BMD has some power to predict fractures (9). Therefore, prospective, randomized, long-term ( $>12 \mathrm{mo}$ ) controlled trials using clinically relevant doses of ICS with adjustment for confounders and with BMD as an endpoint seem to be the best way to assess the risk of clinically important adverse effects of ICS on the bone (8).

There are a few prospective, randomized, long-term $(\geq 12$ mo) controlled clinical trials assessing the effects of ICS on bone in children (10-13). Two cross-sectional database reviews have assessed fracture risk in children treated with ICS $(14,15)$. Both groups concluded that ICS use does not increase the risk of fractures in children. However, the reviewed studies included both prepubertal and pubertal children up to the age of $17 \mathrm{y}$, which may have caused confounding results.

An observational study in asthmatic children receiving budesonide (BUD) for 3-6 y did not show differences in BMD compared with control children (16). A decade later, the same authors reported that treatment with BUD at a mean daily dose of $350 \mu \mathrm{g}$ for a mean of $14 \mathrm{y}$ (mean accumulated dose, 1775 $\mathrm{mg}$; range, 227-8760 $\mathrm{mg}$ ) did not adversely affect BMD in adulthood in these children with asthma (Pedersen S, Agertoft L 2009 Peak bone mineral density (BMD) in children with asthma treated for a mean of $14 \mathrm{y}$ with inhaled budesonide. 2009 European Respiratory Society Congress, 12-16 September, 2009, Vienna, Austria, Abstract P1212). Pubertal development with its burst of anabolic steroids is considered a major biologic factor promoting bone formation.

We have previously reported the effects of continuous and periodical use of inhaled BUD on growth of prepubertal children

Abbreviations: BMD, bone mineral density; BMC, bone mineral content; BUD, budesonide; CV, coefficient of variation; DSCG, disodium cromoglycate; DXA, dual energy x-ray absorptiometry; ICS, inhaled corticosteroid; ICTP, carboxyterminal telopeptide of type I procollagen; PINP, aminoterminal propeptide of type I procollagen; SGA, small for GA 
with mild, persistent asthma (17). Continuous BUD was associated with fewer exacerbations and better asthma control. Standing height velocity was normalized during low-dose BUD treatment within $1 \mathrm{y}$ of commencement of treatment. However, the height velocity increased more rapidly during periodical use of BUD than during the low-dose BUD treatment, suggesting catch-up of the initial loss in standing height.

As a secondary part of this same study, we here report results on BMD and markers of bone metabolism in relation to growth.

\section{METHODS}

Subjects. Children aged 5-10 y with newly detected mild asthma participated in a partly double-blind, randomized, parallel-group, single-center 18 -mo study (17). Children with chronic diseases other than asthma that could influence the performance of the study or interpretation of study results were excluded. Children with a history of inhaled, nasal or oral corticosteroid use during the last 2 mo before the study were also excluded. The total cumulative doses of previously used ICS must not have exceeded $36 \mathrm{mg}, 12 \mathrm{mg}$ of nasal corticosteroids or oral doses equivalent to $200 \mathrm{mg}$ of prednisolone. Children not in Tanner stage I at first visit and in stage $>$ II at the end of the study as well as SGA children without a catch-up growth within 2 y of life were excluded in the BMD and growth analysis.

Study design and treatment. Children were randomized in balanced blocks into three treatment groups, two double-blind arms, and one open-treatment arm: 1) continuous budesonide (BUD/BUD, $400 \mu \mathrm{g}$ twice daily for $1 \mathrm{mo}, 200$ $\mu \mathrm{g}$ twice daily for $2-6 \mathrm{mo}, 100 \mu \mathrm{g}$ twice daily for 7-18 mo; 2) budesonide/ placebo (BUD/PLA), identical treatment as in Group 1 during months 1-6, but thereafter placebo for 7-18 mo; and 3) disodium cromoglycate (DSCG) $10 \mathrm{mg}$ three times daily for $1-18 \mathrm{mo}$. The two BUD treatments were double-blinded in administration; DSCG was given by open label. BUD was administered via the dry powder inhaler, Turbuhaler, and DSCG via a pressurized metered dose inhaler. Exacerbations in all three groups were treated with BUD $400 \mu \mathrm{g}$ twice daily for 2 -wk period. This means that children in the BUD/PLA group received only periodic treatment with BUD during months $7-18$ in case of an asthma worsening. Children with frequent exacerbations (frequent defined as a time interval of $\leq 2$ mo between two exacerbations) could in addition receive oral theophylline (Theo-Dur, AstraZeneca, Södertälje, Sweden) for $6 \mathrm{wk}$ in a daily dose of $10-15 \mathrm{mg} / \mathrm{kg}$ body weight. If this additional therapy was insufficient, the children received treatment with oral prednisolone and were excluded from the study.

Assessments. Each morning and evening throughout the study, the children measured their peak expiratory flow (PEF) and the peak inspiratory flow through the inhaler using a portable data storage spirometer (Vitalograph Ltd, Buckingham, UK) (17). Thereby adherence to twice daily treatment could be recorded.

At baseline and after treatment for $18 \mathrm{mo}$, the patients had measurement of BMD. Height was recorded before treatment and thereafter every third month with a stadiometer (Holtain Ltd, Crymych, UK) following a standardized procedure. The median value of three measurements was recorded. Tanner stage (sexual maturation) was scored at enrolment and at the end of the study.

BMD was assessed by dual energy x-ray absorptiometry (DXA), in lumbar vertebrae 1-4 using Hologic QDR 1000 densitometer (Hologic Inc., Waltham, MA) (18). Only one radiologist performed and analyzed all DXA measurements. A single device at the University Central Hospital of Helsinki was used. Quality control was maintained by daily scanning of an anthropomorphic spine phantom. The coefficient of variation (CV) for the DXA technique in adults has been reported to be $0.9 \%$ for lumbar spine (19). In children, the $\mathrm{CV}$ was not determined, because it was considered unethical to repeatedly expose them to $\mathrm{x}$-rays. The unit contains a bottom-mounted $\mathrm{x}$-ray source that releases a tightly collimated photon beam of altering energy (70 and $140 \mathrm{keV}$ ) that passes sequentially from the source through an internal reference standard and through the patient to a detector. Children were scanned in supine position during 5-8 min including 2-4 min of radiation time. Projected area $\left(\mathrm{cm}^{2}\right)$ and bone mineral content (BMC, i.e. grams of hydroxyapatite) were measured for vertebrae L-1 through L-4. The BMC value was divided by the surface area for each vertebra giving its BMD [grams per square centimeter $\left(\mathrm{g} / \mathrm{cm}^{2}\right)$ ]. The mean value of the four vertebrae gave the BMD of each child. BMD was also expressed as Z-scores.

At baseline and after treatment for 6,12 , and $18 \mathrm{mo}$, blood and urine samples were collected for determination of markers of bone formation and degradation, i.e. serum osteocalcin (20) and aminoterminal propeptide of type I procollagen (PINP) (21) as markers of bone formation and carboxyterminal telopeptide of type I procollagen (ICTP) (22) and urinary deoxypyridinoline (23) as markers of bone resorption. The urinary result was corrected by dividing deoxypyridinoline $(\mathrm{nmol} / \mathrm{L})$ with creatinine concentration $(\mathrm{mmol})$ of the sample and expressed as U-deoxypyridinoline/creatinine. The intra and interassay CVs were $4.5-6.2 \%$ and $4.1-8.9 \%$ for serum osteocalcin, $2.4-$ $3.5 \%$ and $2.7-6.7 \%$ for serum PINP, $3.8-7.3 \%$ and $2.5-8.0 \%$ for serum ICTP, and $1.4-5.3 \%$ and $7.3-14.2 \%$ for urinary deoxypyridinoline (24).

The study was performed in accordance with the Declaration of Helsinki and Good Clinical Practice (GCP) and was approved by the local Ethics Committee. Written informed consent was obtained from each patient's parent(s) or legal guardian and from the patient.

Statistical analysis. The sample size was determined by power calculations for morning PEF rates (17). The changes in BMD and height from baseline to end of treatment were compared between the three treatment regimens with an additive ANOVA model with fixed factors treatment and sex and using baseline as a covariate. Clinical chemistry and urine variables were compared between treatments using a similar but multiplicative ANOVA model, i.e. data were log transformed before analysis. The ratio of changes in BMD over changes in height was compared between treatments with an additive ANOVA model with fixed factors treatment and sex. Here, the changes are expressed as changes per $\mathrm{cm}$ grown.

Comparisons for months $1-6$ were made between the combined BUD groups and the DSCG group; comparisons at 12 and 18 mo were made between the three groups. All tests were two-sided, and $p$ values $<0.05$ were considered statistically significant.

\section{RESULTS}

Patient demographics. The baseline characteristics of the study population are shown in Table 1. Based on symptoms and lung function tests, the majority of the patients had mild persistent asthma (1). Only a few children had used ICS earlier in life and nobody during the 2-mo period preceding study entry.

Performance of the study. Figure 1 shows the flow of the patients during the study. A total of 178 children were randomized and 176 received allocated treatment. Of these, 142 had BMD measurements before and after the study. Patients withdrawn from the study (Fig. 1) did not have BMD measurements at the time of withdrawal. The primary analysis of growth and bone used this completer population not fulfilling some prespecified exclusion criteria; i.e. children with a birth length less than -2 SD and with no catch-up within the first 2 years, children with Tanner stage II at entry or Tanner stage III at the last visit. Six such children were excluded, two in each treatment group. Two additional children had one missing value each on BMD. The analysis population thus consisted of 136 children. Table 2 shows the mean doses of BUD used in the three treatment groups. A total of 364 exacerbations in 133 children were treated with 2-wk courses of BUD: 81 in the BUD/BUD group, 121 in the BUD/PLA group, and 162 in the DSCG group. Three exacerbations in three children

Table 1. Baseline characteristics of the patients

\begin{tabular}{|c|c|c|c|}
\hline Treatment group & $\begin{array}{l}\text { Continuous } \\
\text { budesonide }\end{array}$ & $\begin{array}{c}\text { Budesonide/ } \\
\text { placebo (periodic } \\
\text { budesonide) }\end{array}$ & $\begin{array}{c}\text { Disodium } \\
\text { cromoglycate }\end{array}$ \\
\hline No. of patients & 50 & 44 & 42 \\
\hline Age (yrs) & $6.9(5-10)$ & $6.7(5-9)$ & $7.0(5-10)$ \\
\hline Male $(\%)$ & 60 & 66 & 50 \\
\hline $\operatorname{BMD}\left(\mathrm{g} / \mathrm{cm}^{2}\right)$ & $0.62(0.50-0.77)$ & $0.61(0.53-0.74)$ & $0.61(0.45-0.76)$ \\
\hline BMC (g) & $19.4(12-33)$ & $18.3(13-26)$ & $19.0(10-32)$ \\
\hline Bone area $\left(\mathrm{cm}^{2}\right)$ & $31(22-47)$ & $30(22-41)$ & $31(20-45)$ \\
\hline Height $(\mathrm{cm})$ & $129(110-158)$ & $125(106-143)$ & $127(107-147)$ \\
\hline Weight $(\mathrm{kg})$ & $29(19-51)$ & $27(17-49)$ & $28(16-52)$ \\
\hline
\end{tabular}

Values are means with range in parentheses, unless otherwise stated. 


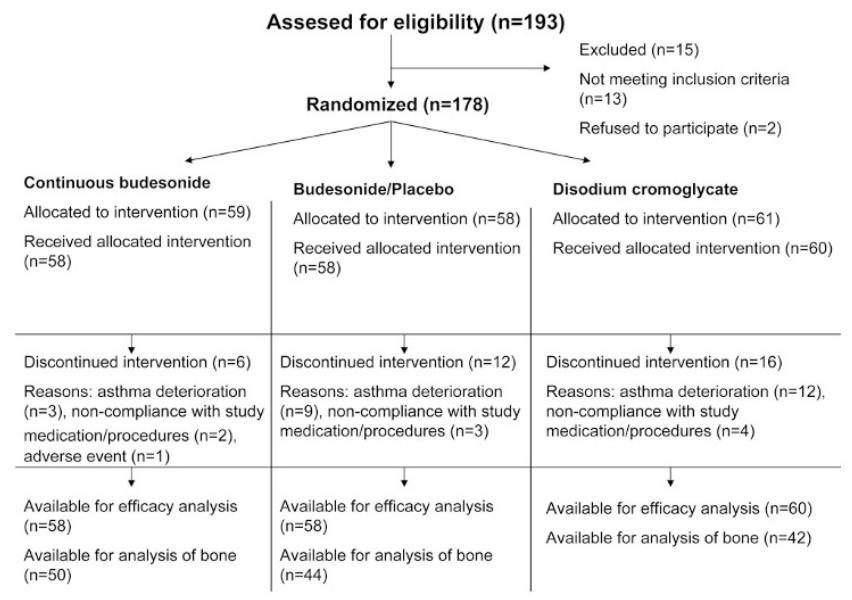

Figure 1. Flow chart of the patients.

Table 2. Mean daily doses of budesonide, $\mu g /$ day (range), during the study

\begin{tabular}{cccc}
\hline Period & $\begin{array}{c}\text { Continuous } \\
\text { budesonide }\end{array}$ & $\begin{array}{c}\text { Budesonide/ } \\
\text { placebo }\end{array}$ & $\begin{array}{c}\text { Disodium } \\
\text { cromoglycate }\end{array}$ \\
\hline $\begin{array}{c}\text { 0- to 6-mo } \\
\text { period }\end{array}$ & $468.5(454.2-800.0)$ & $468.5(454.2-800.0)$ & $63.3(0.0-417.6)$ \\
$\begin{array}{c}\text { 7- to 18-mo } \\
\text { period }\end{array}$ & $223.4(200.0-423.8)$ & $32.6(0.0-487.0)$ & $62.6(0.0-288.9)$ \\
\hline
\end{tabular}

had to be treated with oral steroids, and these children were withdrawn from the study. Adherence to treatment, based on the data storage spirometer results, was $\sim 90 \%$ in the beginning of the study but decreased to a level of $\sim 60 \%$ by the end of the study.

Bone mineral density. The results of the statistical analysis of changes in BMD given both as $\mathrm{g} / \mathrm{cm}^{2}$ and as Z-scores are shown in Table 3. A statistically significantly larger increase in BMD was found for both parameters in the DSCG than in the BUD/BUD group. The increase in BMD was intermediate in the BUD/PLA group. Calculated as grams per square meter, the mean increment in BMD was 3.7\% in the BUD/BUD group, $4.8 \%$ in the BUD/PLA group, and $5.5 \%$ in the DSCG group. The mean increments in BMC were 14.4, 14.9, and $16.2 \%$ (no significant differences between the groups) and 10.4, 9.4, and $10.3 \%$ in bone area (no significant differences between the groups) in the respective treatment groups.

After treatment for $6 \mathrm{mo}$, there was a statistically significant difference in changes in height between BUD-treated (combined group) and DSCG-treated children. After 12 and 18 mo, there were significant differences between all three groups when compared two by two.
At baseline, there was a statistically significant correlation between BMD and height ( $n=175 ; r=0.325 ; p<0.001$ ). There was also a statistically significant correlation between BMD and height for the 136 BUD-treated patients during the study $(r=0.333 ; 95 \%$ CI $0.173-0.475 ; p<0.001)$. The increase in BMD adjusted for the increase in height showed no significant differences between the groups (Table 4). This suggests that the differences in change in BMD could be explained by the change in growth. Numerically the ratio (slope) was smallest in the BUD/BUD group. No significant differences in body mass index were observed between treatment groups at any time point. The results of the clinical chemistry measurements are shown in Figure 2. Statistically significant differences between the combined BUD group and the DSCG group were seen on all four parameters after the 6-mo treatment. No statistically significant differences were found at the end of the study except between BUD/BUD and DSCG on serum ICTP ( $p=0.036$; Fig. 2).

\section{DISCUSSION}

This study reporting BMD in children with asthma is part of a previously reported, double-blind, randomized, 18-mo study that compared regular and periodical BUD treatment strategies (18). Regular use of high-dose BUD followed by low dose afforded better asthma control and fewer exacerbations than periodic use of BUD given only at times of exacerbations. Standing height velocity was normalized during lowdose BUD treatment. The height velocity increased, however, more rapidly during periodical use of BUD than during the low-dose BUD treatment, suggesting catch-up of the initial loss in standing height (17). The effects we report on BMD in this secondary analysis are the result of BUD treatment because only three patients required oral steroids for exacerbations. Consequently, in this study, use of oral steroids cannot have influenced the BMD results of children with worsening asthma. A limitation of our study is, however, that it only lasted for 18 mo. In a long-term study, the number of exacerbation requiring high-dose BUD might have increased. The main reason for not prolonging the study period was to avoid any influence of puberty on BMD and height.

During the study, BMD increased in all treatment groups. However, the regular use of budesonide (BUD/BUD) resulted at the end of the study in a statistically significantly smaller increment in BMD than DSCG calculated as $\mathrm{g} / \mathrm{cm}^{2}$ and $Z$ score. The increase in BMD $\left(\mathrm{g} / \mathrm{cm}^{2}\right)$ in the group receiving periodical BUD after the first 6 mo (BUD/PLA group) was not statistically different from the other two groups. The incre-

Table 3. The changes in lumbar bone mineral density during 18 months shown as $\mathrm{g} / \mathrm{cm}^{2}$ and Z-scores in children in the three treatment groups

\begin{tabular}{|c|c|c|c|c|c|c|c|}
\hline \multirow[b]{2}{*}{ Treatment comparisons } & \multirow[b]{2}{*}{$N$} & \multicolumn{3}{|c|}{$\Delta \mathrm{BMD}\left(\mathrm{g} / \mathrm{cm}^{2}\right)$} & \multicolumn{3}{|c|}{$\Delta Z$-score } \\
\hline & & $\Delta$ & $95 \% \mathrm{CI}$ & $p$ & $\Delta$ & $95 \% \mathrm{CI}$ & $p$ \\
\hline BUD/BUD & 50 & 0.023 & $0.017,0.029$ & & -0.29 & $-0.38,-0.20$ & \\
\hline BUD/PLA & 44 & 0.029 & $0.022,0.036$ & & -0.16 & $-0.26,-0.06$ & \\
\hline DSCG & 42 & 0.034 & $0.027,0.041$ & & -0.13 & $-0.23,-0.04$ & \\
\hline B/BUD-B/PLA & & -0.006 & $-0.015,0.003$ & 0.200 & -0.13 & $-0.26,0.00$ & 0.052 \\
\hline B/BUD-DSCG & & -0.011 & $-0.020,-0.002$ & 0.022 & -0.16 & $-0.29,-0.02$ & 0.021 \\
\hline B/PLA-DSCG & & -0.005 & $-0.014,0.005$ & 0.319 & -0.03 & $-0.17,0.11$ & 0.700 \\
\hline
\end{tabular}


Table 4. The increment in height and in BMD adjusted by the increment in height

\begin{tabular}{|c|c|c|c|c|c|c|c|}
\hline \multirow[b]{2}{*}{ Treatment comparisons } & \multirow[b]{2}{*}{$N$} & \multicolumn{3}{|c|}{$\Delta$ Height $(\mathrm{cm})$} & \multicolumn{3}{|c|}{$\Delta \mathrm{BMD} / \Delta$ Height $\left(1000 \mathrm{~g} / \mathrm{cm}^{3}\right)$} \\
\hline & & $\Delta$ & $95 \% \mathrm{CI}$ & $P$ & $\Delta$ & $95 \% \mathrm{CI}$ & $p$ \\
\hline BUD/BUD & 50 & 7.75 & $7.34,8.17$ & & 2.99 & $2.25,3.73$ & \\
\hline BUD/PLA & 44 & 8.18 & $7.73,8.63$ & & 3.59 & $2.80,4.39$ & \\
\hline DSCG & 42 & 8.80 & $8.35,9.25$ & & 3.75 & $2.95,4.55$ & \\
\hline B/BUD-B/PLA & & -0.42 & $-1.03,0.18$ & 0.170 & -0.60 & $-1.68,0.47$ & 0.271 \\
\hline B/BUD-DSCG & & -1.05 & $-1.66,-0.44$ & 0.001 & -0.76 & $-1.85,0.33$ & 0.172 \\
\hline B/PLA-DSCG & & -0.63 & $-1.26,0.01$ & 0.053 & -0.16 & $-1.29,0.97$ & 0.784 \\
\hline
\end{tabular}
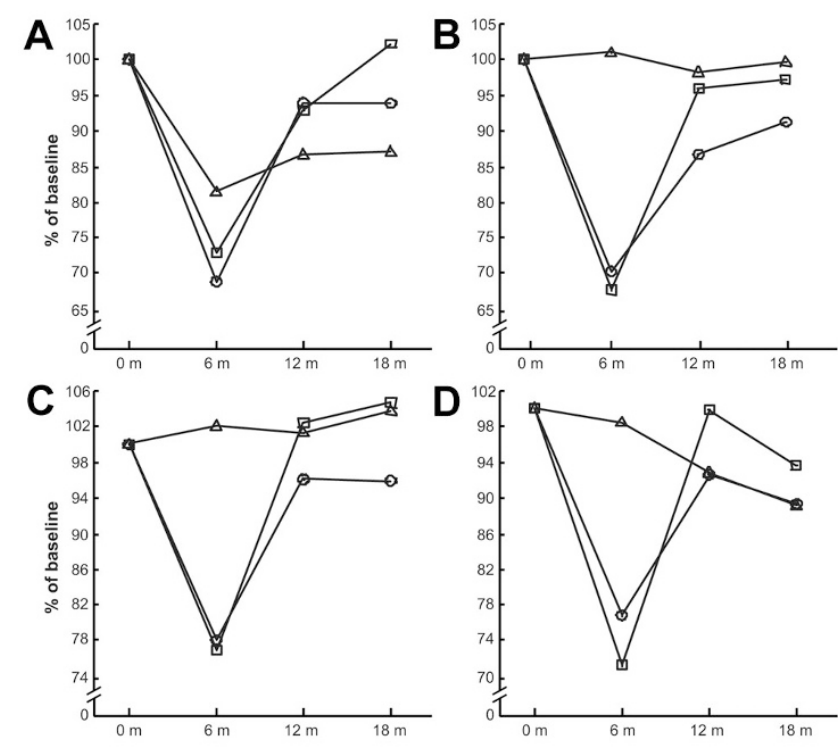

Figure 2. Changes in serum osteocalcin $(A)$, serum PIPN $(B)$, serum ICTP $(C)$, and urinary deoxypridinoline $(D)$ in three treatment groups, $\bigcirc$, BUD/ BUD; $\square$, BUD/placebo; and $\triangle$, DSCG. Statistically significant differences between the combined BUD group and the DSCG group were seen on all four parameters after the 6-mo treatment.

ments in mean BMC resemble the changes in BMD between the groups although these changes were not statistically significant. Instead, the increments in mean bone areas were on the same level between the groups suggesting that BMC might be the driver of the differences in BMD. When corrected for growth data, the differences in BMD data disappeared. Children discontinuing the study prematurely did not have BMD measurements when withdrawn from the study.

After high-to-moderate dose BUD treatment for $6 \mathrm{mo}$, all four markers of bone metabolism were significantly reduced compared with the DSCG group. At the end of the study, when BUD had been administered in a low dose or periodically, no significant differences were observed between the groups in bone formation markers; serum PINP and osteocalcin. ICTP, the serum marker of bone resorption, was slightly lower in children receiving BUD regularly compared with the DSCG group. However, no significant differences were observed in urine deoxypyridinoline, another marker of bone resorption. Therefore, this finding of decreased ICTP remains unclear. These findings in markers of bone metabolism describe the actual bone turnover after the 18-mo treatment and not the net results in BMD and standing height. The rapid increase in the levels of bone markers during the periodic and regular low-dose BUD suggests a tendency toward normal age dependent bone metabolism.

The nonsignificant ICS effect on BMD in our study when corrected for growth data are in accordance with a previous report in 49 children treated with beclomethasone dipropionate or DSCG for $7 \mathrm{mo}$ in an open, nonrandomized study (25). When BMD was adjusted for body size, bone mass was found not to have changed after either treatment within or between the groups. It thus appears that following height velocity of children with asthma treated with ICS is good enough for following the risk for adverse effects during longterm treatment with ICS. As previously shown, BUD had a dose-dependent effect on linear growth in the study (17).

The Childhood Asthma Management Program (CAMP) comparing the effects of BUD $400 \mu \mathrm{g} / \mathrm{d}$ with nedocromil 16 $\mathrm{mg} / \mathrm{d}$ over $4-6 \mathrm{y}$ in $5-12-\mathrm{y}$-old children $(n=1041)$ did not find a difference in BMD between the groups (13). A crosssectional analysis of baseline data in the CAMP study found no significant effect of previous ICS use on BMD (26). The analysis of the CAMP data (13) was, however, an intentionto-treat analysis that would not have identified outliers with low rates of bone accretion. Some children also reached puberty during the study. The CAMP protocol also allowed use of prednisone bursts for exacerbations and introduction of open label ICS for worsening asthma. Multiple oral steroid bursts were found to produce a dose-dependent reduction in BMC and increased risk for osteopenia. ICS use had the potential for reducing bone mineral accretion in boys progressing through puberty, but this risk was considered to be outweighed by the ability to reduce the amount of oral steroids used in these children (27).

To the best of our knowledge, this study is the second long-term randomized, double-blind study evaluating the effects of high-dose ICS, followed by low-dose ICS in prepubertal children. The results in this study could further be compared with periodical ICS therapy and nonsteroidal therapy (DSCG). Our study protocol therefore differs in several aspects from that of the first long-term study reported by Visser et al. (12). They did not found differences in BMD between the two fluticasone propionate regimens but no comparison with healthy controls or asthmatic children not receiving ICS therapy were included. The results are therefore difficult to compare.

Markers of both bone formation and bone resorption can be easily measured and several studies have evaluated the influence of ICS on them $(4-7,15,26-28)$. Overall, the results have been reassuring indicating minimal effects of ICS. Doses of ICS 
producing good asthma control in the majority of patients have not been associated with detectable effects on these markers (29) whereas subtle changes have sometimes been reported with high dose ICS. As an example, Boot et al. (30) did not find differences in mean osteocalcin, PICP, and ICTP levels between asthmatic children treated with ICS and controls. In contrast, Akil et al. (28) found a significant decrease in osteocalcin and increase in ICTP levels compared with pretreatment values in a study in 22 asthmatic children. Also, in the study comparing high-dose with low-dose fluticasone propionate, significant initial reductions in osteocalcin and PINP levels were noticed without a difference in effect on BMD (12). In our study, high-dose BUD for 6 mo resulted in a clear decrease in both markers of bone formation and degradation. The changes were much larger than the intra and interassay CVs. During subsequent low-dose treatment, all mean values, except ICTP, returned to baseline. Complete normalization occurred in the group receiving periodic treatment with BUD. In simple terms, an elevation of all markers could occur when there is increased bone turnover without net loss or gain in bone mass, whereas a reduction of all markers, which is normally seen with low doses of oral corticosteroids or high doses of ICS, could signify a reduction in bone turnover leading to a reduction of the age-dependent increase in BMD and standing height. It is also clinically more relevant to consider the net effect of bone formation and bone resorption (31). When markers of formation and resorption, as in our study, decrease to the same extent, a real bone loss may possibly not occur.

Our findings suggest that inhaled BUD may induce a slight but statistically significant decrease in BMD in prepubertal children with asthma. This effect seems to be linked to the 6-mo initial treatment with high to moderate doses of BUD. The effect was, however, still measurable after 1 y of treatment with low-dose BUD. As a correlation exists, although not applicable on an individual level, between BMD and risk of fractures, we believe that our findings related to BMD are of clinical importance. The observed decrease in height velocity was in line with the changes in BMD. Between periodic BUD and DSCG treatments, no significant differences in decrease were observed, neither for BMD nor for standing height. The correlation between height and BMD suggests that following children's height might afford an estimation of ICS effects on bone. A clinically important observation is that treatment with ICS should always aim at the lowest effective dose that keeps the patient in good asthma control, thereby the risk of adverse events is minimized.

Acknowledgments. We thank the children and their families for their excellent compliance during this long-term study. We also acknowledge the valuable contribution of Tuula Koljonen, RN, and Leena Ingelin-Kuortti, RN, study nurses; Eeva Kiiskilä and Eva Holtås, study monitors; Thomas Bengtsson, MSc, biostatistician; and the radiologist Pekka Mäkelä, MD, PhD, for the DXA measurements.

\section{REFERENCES}

1. National Institutes of Health. NHLBI/WHO Workshop report: Global strategy for asthma management and prevention. National Institutes of Health, National Heart,
Lung and Blood Institute, Washington, DC. Available at: www.ginasthma.com. Accessed September 10, 2009

2. Allen DB, Mullen M, Mullen B 1994 A meta-analysis of the effect of oral and inhaled corticosteroids on growth. J Allergy Clin Immunol 93:967-976

3. van Staa T-P, Cooper PM, Leufkens HG, Bishop N 2003 Children and the risk of fractures caused by oral corticosteroids. J Bone Miner Res 18:913-918

4. Hubbard RB, Smith CJ, Smeeth L, Harrison TW, Tattersfield AE 2002 Inhaled corticosteroids and hip fracture. Am J Respir Crit Care Med 166:1563-1566

5. Suissa S, Baltzan M, Kremer R, Ernst P 2003 Inhaled and nasal corticosteroid use and the risk of fracture. Am J Respir Crit Care Med 169:83-88

6. Mortimer KJ, Harrison TW, Tattersfield AE 2005 Effects of inhaled corticosteroids on bone. Ann Allergy Asthma Immunol 94:15-22

7. Jones A, Fay JK, Burr M, Stone M, Hood K, Roberts G 2002 Inhaled corticosteroid effects on bone metabolism in asthma and mild chronic obstructive pulmonary disease. Cochrane Database Syst Rev 1:CD003537

8. Pedersen S 2006 Clinical safety of inhaled corticosteroids for asthma in children. An update of long-term trials. Drug Saf 29:599-612

9. Marshall D, Johnell O, Wedel H 1996 Meta-analysis of how well measures of bone mineral density predict occurrence of osteoporotic fractures. BMJ 312:1254-1259

10. Gregson RK, Rao R, Murrills AJ, Taylor PA, Warner JO 1998 Effect of inhaled corticosteroids on bone mineral density in childhood asthma: comparison of fluticasone propionate with beclomethasone dipropionate. Osteoporos Int 8:418-422

11. Roux C, Kolta S, Desfougéres JL, Minini P, Bidat E 2003 Long-term safety of fluticasone propionate and nedocromil sodium on bone in children with asthma. Pediatrics 111:e706-e713

12. Visser MJ, van der Veer E, Postma DS, Arends LR, de Vries TW, Brand PL, Duiveman EJ 2004 Side-effects of fluticasone in asthmatic children: no effects after dose reduction. Eur Respir J 24:420-425

13. Szefler S, Weiss S, Tonascia J 2000 Long-term effects of budesonide or nedocromil in children with asthma. The Childhood Asthma Management Program Research Group. N Engl J Med 343:1054-1063

14. Schlienger RG, Jick SS, Meier CR 2004 Inhaled corticosteroids and the risk of fractures in children and adolescents. Pediatrics 114:469-473

15. van Staa T-P, Bishop N, Leufkens HG, Cooper C 2004 Are inhaled corticosteroids associated with an increased risk of fracture in children? Osteoporos Int 15:785-791

16. Agertoft L, Pedersen S 1998 Bone mineral density in children with asthma receiving long-term treatment with inhaled budesonide. Am J Respir Crit Care Med 157:178-183

17. Turpeinen M, Nikander K, Pelkonen AS, Syvänen P, Sorva R, Raitio H, Malmberg P, Juntunen-Backman K, Haahtela T 2008 Daily versus as-needed inhaled corticosteroid for mild persistent asthma. The Helsinki Early Intervention Childhood Asthma Study. Arch Dis Child 93:654-659

18. Southard RN, Morris JD, Mahan JD, Hayes JR, Torch MA, Sommer A, Zipf WB 1991 Bone mass in healthy children: measurements with quantitative DXA. Radiology 179:735-738

19. Välimäki MJ, Kinnunen K, Volin L, Tähtelä R, Löyttyniemi E, Laitinen K, Mäkelä P, Keto P, Ruutu T 1999 A prospective study of bone loss and turnover after allogeneic bone marrow transplantation: effect of calcium supplementation with or without calcitonin. Bone Marrow Transplant 23:355-361

20. Garnero P, Grimaux M, Seguin P, Delmas PD 1994 Characterization of immunoreactive forms of human osteocalcin generated in vivo and in vitro. $\mathrm{J}$ Bone Miner Res 9:255-264

21. Risteli L, Risteli J 1993 Biochemical markers of bone metabolism. Ann Med 25:385-393

22. Risteli J, Elomaa I, Niemi S, Novamo A, Risteli L 1993 Radioimmunoassay for pyridinoline cross-linked carboxyterminal telopeptide of type I collagen: a new serum marker of bone collagen degradation. Clin Chem 39:635-640

23. Delmas PD, Schlemmer A, Ginetys E, Riis B, Christiansen C 1991 urinary excretion of pyridoline cross-links correlates with bone turnover measured in iliac crest biopsy in patients with vertebral osteoporosis. J Bone Miner Res 6:639-644

24. Tähtelä R, Turpeinen M, Sorva R, Karonen SL 1997 The aminoterminal propeptide of type I procollagen: evaluation of a commercial radioimmunoassay kit and values in healthy subjects. Clin Biochem 30:35-40; erratum in Clin Biochem 30:241

25. Martinati LC, Bertoldo F, Gasperi E, Fortunati P, Lo Cascio V, Boner AL 1998 Longitudinal evaluation of bone mass in asthmatic children treated with inhaled beclomethasone dipropionate or cromolyn sodium. Allergy 53:705-708

26. Kelly HW, Strunk RC, Donithan M, Bloomberg GR, McWilliams BC, Szefler S 2003 Growth and bone density in children with mild-moderate asthma: a crosssectional study in children entering the Childhood Asthma Management Program (CAMP). J Pediatr 142:286-291

27. Kelly HW, Van Natta ML, Covar RA, Tonascia J, Green RP, Strunk RC. CAMP Research Group 2008 Effect of long-term corticosteroid use on bone mineral density in children: a prospective longitudinal assessment in the childhood asthma management program (CAMP) study. Pediatrics 122:e53-e61

28. Akil I, Yüksel H, Ürk V, Var A, Onur E 2004 Biochemical markers of bone metabolism and calciuria with inhaled budesonide therapy. Pediatr Nephrol 19:511-515

29. Chay OM, Goh A, Lim WH, Leong KH, Lou J 1999 Effects of inhaled corticosteroid on bone turnover in children with bronchial asthma. Respirology 4:63-67

30. Boot AM, de Jongste JC, Verberne AA, Pols HA, de Muinck Keizer-Schrama SM 1997 Bone mineral density and bone metabolism of prepubergyal children with asthma after long-term treatment with inhaled corticosteroids. Pediatr Pulmonol 24:379-384

31. Pedersen S 2001 Do inhaled corticosteroids inhibit growth in children? Am J Respir Crit Care Med 164:521-535 